\title{
REFERENCES
}

1. V. F. Cowling, Walter Leighton and W. J. Thron, Twin convergence regions for continued fractions, Bull. Amer. Math. Soc. 50 (1944), 351-357.

2. R. E. Lane, Absolute convergence of continued fractions, Proc. Amer. Math. Soc. 3 (1952), 904-913.

3. R. E. Lane and H. S. Wall, Continued fractions with absolutely convergent even and odd parts, Trans. Amer. Math. Soc. 67 (1949), 368-380.

4. H. S. Wall, Some convergence problems for continued fractions, Amer. Math. Monthly 64 (1957), 95-103.

UNIVERSITY OF TEXAS AND

UNIVERSITY OF KENTUCKY

\section{NOTE ON A NONLINEAR EIGENVALUE PROBLEM}

\section{MARVIN SHINBROT ${ }^{1}$}

1. In the theory of hydrodynamic stability, eigenvalue problems of the form

$$
L u+\frac{1}{\lambda} M u=\lambda u
$$

arise $[1$, p. 430$]$. Here, $L$ and $M$ denote ordinary differential operators, the order of $L$ exceeds that of $M$, and the boundary conditions are such that $L$ is self-adjoint. One of the questions of interest is whether there exist eigenvalues of this problem and, if so, whether the corresponding eigenfunctions are complete.

Replacing $\lambda$ by $1 / \lambda$, it is easy to see that if $L^{-1}$ exists, (1.1) is equivalent to

$$
\lambda u=A u+\lambda^{2} B u,
$$

where $A=L^{-1}$ and $B=-L^{-1} M$ are compact, and $A$ is symmetric. In this note, we shall consider the question of the completeness of the eigenfunctions of the following generalization of (1.2):

$$
\lambda u=A u+\lambda^{\alpha} B_{\lambda} u,
$$

where $\alpha>1, A$ is compact and symmetric, and $B_{\lambda}$, which, as the notation indicates, may depend on $\lambda$, is merely bounded. More precise

Received by the editors May 11, 1962.

1 The work reported here was supported by the United States Air Force under Grant AF-AFOSR-62-136. 
hypotheses on $A$ and $B$ will be specified below.

2. We shall operate entirely in a Hilbert space $H$. The symbols $\mu_{n}$ and $v_{n}$ will be used consistently to denote the eigenvalues and corresponding eigenfunctions of $A$, in the usual order and with the usual normalization.

The subspace of $H$ spanned by all but the first $N-1$ eigenfunctions of $A$ will be denoted by $H_{N}: H_{N}=\left\{v_{N}, v_{N+1}, \cdots\right\}$. If infinitely many eigenfunctions of (1.3) exist, the space spanned by all but the first $N-1$ of them will be denoted by $K_{N}$.

It will be assumed that $A$ satisfies the hypothesis

(a). A is a compact, symmetric operator with simple eigenvalues $\mu_{n}$. Its eigenfunctions $v_{n}$ span $H$. Moreover, if

$$
\delta_{n}=\min _{j \neq n}\left|\mu_{n}-\mu_{j}\right|,
$$

then

$$
\sum\left|\frac{\mu_{n}^{\alpha}}{\delta_{n}}\right|^{2}<\infty .
$$

The assumptions on $B_{\lambda}$ are set forth in

(B). $B_{\lambda}$ is an operator defined for all values of the parameter $\lambda$ in the disk

$$
\lambda \leqq \frac{\alpha}{\alpha-1}\|A\| .
$$

$B_{\lambda}$ is uniformly bounded (by $\|B\|$, say) in the disk (2.3) and satisfies a Lipschitz condition

$$
\left\|B_{\lambda}-B_{\mu}\right\| \leqq L|\lambda-\mu|
$$

there.

We shall prove the following

THEOREM. Let (Q) and (B) be satisfied and let $\alpha$ exceed unity. Then, there exist infinitely many eigenvalues $\lambda_{n}$ and eigenfunctions $u_{n}$ of (1.3). The sequence $\left\{\lambda_{n}\right\}$ approaches zero. Moreover, there is an integer $N$ such that $K_{N} \supset H_{N}$ and, if $x$ is any element of $H$, it can be represented in the form

$$
x=\sum_{1}^{N-1} \xi_{n} v_{n}+\sum_{N}^{\infty} \xi_{n} u_{n} .
$$

Finally, if $\|A\|$ and the series (2.2) are small enough, ${ }^{2} N$ may be

: The assumption on $\|A\|$ cannot be eliminated by normalization since the required size of $\|A\|$ depends on $\|B\|$ in just such a way that the normalization constant cancels out. 
taken as unity, so that the sequence $\left\{u_{n}\right\}$ is complete in $H$.

Before proving this theorem, let us note here once and for all that the letter $c$, with or without subscripts, will always be used to denote a constant independent of $n$. Generally, it will denote different constants in different formulas.

3. It is easy to prove the existence of a sequence of real eigenvalues approaching zero, even if (2.2) is violated, if $B_{\lambda}$ is compact and symmetric and if

$$
\|B\| \cdot\|A\|^{\alpha-1} \leqq \frac{(\alpha-1)^{\alpha-1}}{\alpha^{\alpha}}
$$

Define a mapping of the interval (2.3) into the real line as follows. Choose a value of $\lambda$ in (2.3) and, for any fixed $n$, let $\lambda^{\prime}$ denote the $n$th eigenvalue of the operator $A_{\lambda}=A+\lambda^{\alpha} B_{\lambda}$. Note that $\lambda^{\prime}$ exists since $\lambda$ is fixed, while $A_{\lambda}$ is compact and symmetric and does not depend on $\lambda^{\prime}$. Also,

$$
\left|\lambda^{\prime}\right| \leqq\left\|A_{\lambda}\right\| \leqq\|A\|+|\lambda|^{\alpha}\|B\| \leqq \frac{\alpha}{\alpha-1}\|A\|,
$$

by (3.1) and the choice of $\lambda$. Thus, the mapping takes (2.3) into itself and is also continuous if $B_{\lambda}$ is. For each $n$, then, there exists a fixed point-that is, a value of $\lambda_{n}$ such that $A_{\lambda_{n}}$ has $\lambda_{n}$ as one of its eigenvalues. These $\lambda_{n}$ are obviously eigenvalues of (1.3).

Moreover, $\lambda_{n} \rightarrow 0$, since for each positive value of $\lambda$, however small, all but a finite number of the eigenvalues $\lambda^{\prime}$ are absolutely less than $\lambda$. If $n$ is large enough, then, the mapping just defined takes the interval $[-\lambda, \lambda]$ into itself. Since $\lambda$ was arbitrary, this shows that $\lambda_{n} \rightarrow 0$.

Although the hypothesis (2.2) was not needed in this argument, the assumptions on $B_{\lambda}$ had to be increased. Also, and more important, the method just described does not yield enough information for anything to be said about completeness of the eigenfunctions. To draw the conclusion (2.5), an existence proof that includes certain estimates of $\lambda_{n}$ and $u_{n}$ is required. We now proceed to give such a proof.

Let $b_{\gamma}$ denote the ball of radius $\gamma$ in $H: b_{\gamma}=\{u:\|u\| \leqq \gamma\}$. For some $\gamma$, which will be determined later, select an element $u \in b_{\gamma}$. Fix $n$, and consider the equation

$$
\lambda^{\prime}=\mu_{n}+\lambda^{\alpha}\left(B_{\lambda} u, v_{n}\right)
$$


where $\lambda$ is any number in a disk

$$
|\lambda| \leqq \frac{\alpha}{\alpha-1}\left|\mu_{n}\right|
$$

$B_{\lambda}$ is defined, since $\left|\mu_{n}\right| \leqq\|A\|$ for all $n$. Also,

$$
\left|\lambda^{\prime}\right| \leqq\left|\mu_{n}\right|+\frac{\gamma\|B\| \alpha^{\alpha}}{(\alpha-1)^{\alpha}}\left|\mu_{n}\right|^{\alpha}
$$

so that $\lambda^{\prime}$ satisfies (3.3) if $n$ is large enough.

Since the mapping (3.2) is also continuous, we can define $\lambda_{n}$ as the largest fixed point that satisfies (3.3). For each $u \in b_{\gamma}$, we obtain in this way a sequence $\left\{\lambda_{n}\right\}$ satisfying

$$
\lambda_{n}=\mu_{n}+\lambda_{n}^{\alpha}\left(B_{n} u, v_{n}\right)
$$

(where $B_{n}$ has been written for $B_{\lambda_{n}}$ ) and

$$
\left|\lambda_{n}\right| \leqq c\left|\mu_{n}\right|
$$

Now, define a mapping of $b_{\gamma}$ into $H$ by the equation

$$
u^{\prime}=v_{n}+\sum_{j \neq n} \frac{\lambda_{n}^{\alpha}}{\mu_{n}-\mu_{j}}\left(B_{n} u-\left(B_{n} u, v_{n}\right) u, v_{j}\right) v_{j}
$$

At this point, we determine $\gamma$ so that $b_{\gamma}$ is mapped into itself. We have

$$
\begin{aligned}
\left\|u^{\prime}\right\|^{2} & =1+\sum_{j \neq n}\left|\frac{\lambda_{n}^{\alpha}}{\mu_{n}-\mu_{j}}\left(B_{n} u-\left(B_{n} u, v_{n}\right) u, v_{j}\right)\right|^{2} \\
& \leqq 1+c\left|\frac{\mu_{n}^{\alpha}}{\delta_{n}}\right|^{2} \sum_{j \neq n}\left|\left(B_{n} u-\left(B_{n} u, v_{n}\right) u, v_{j}\right)\right|^{2},
\end{aligned}
$$

by (2.1) and (3.5). Consequently,

$$
\left\|u^{\prime}\right\|^{2} \leqq 1+c\left|\frac{\mu_{n}^{\alpha}}{\delta_{n}}\right|^{2} \cdot\left\|B_{n} u-\left(B_{n} u, v_{n}\right) u\right\|^{2} \leqq 1+c\left|\frac{\mu_{n}^{\alpha}}{\delta_{n}}\right|^{2} \gamma^{2}(1+\gamma)^{2},
$$

where $c$ is not only independent of $n$ but also of $\gamma$. In view of the convergence hypothesis (2.2), we see that for any $\gamma>1$ we can make $\left\|u^{\prime}\right\| \leqq \gamma$ by choosing $n$ large enough.

It will now be shown that (3.6) is a contraction, again if $n$ is large enough. Let $u_{1}$ and $u_{2}$ be any two elements of $b_{\gamma}$, and, suppressing the index $n$ for now, let $\lambda_{1}$ and $\lambda_{2}$ be the corresponding solutions of 
(3.4). Both $\lambda_{1}$ and $\lambda_{2}$ satisfy (3.5), of course. If $u_{1}^{\prime}$ and $u_{2}^{\prime}$ denote the images of $u_{1}$ and $u_{2}$ under (3.6), we have, just as before,

$\left\|u_{2}^{\prime}-u_{1}^{\prime}\right\| \leqq \frac{1}{\delta_{n}}\left\|\lambda_{2}^{\alpha} B_{\lambda_{2}} u_{2}-\lambda_{1}^{\alpha} B_{\lambda_{1}} u_{1}\right\|$

$$
\begin{aligned}
& +\frac{1}{\delta_{n}}\left\|\lambda_{2}^{\alpha}\left(B_{\lambda_{2}} u_{2}, v_{n}\right) u_{2}-\lambda_{1}^{\alpha}\left(B_{\lambda_{1}} u_{1}, v_{n}\right) u_{1}\right\| \\
\leqq & \frac{1}{\delta_{n}}\left\|\lambda_{2}^{\alpha} B_{\lambda_{2}} u_{2}-\lambda_{1}^{\alpha} B_{\lambda_{1}} u_{1}\right\|+\frac{\left|\lambda_{2}\right|^{\alpha}}{\delta_{n}}\left|\left(B_{\lambda_{2}} u_{2}, v_{n}\right)\right| \cdot\left\|u_{2}-u_{1}\right\| \\
& +\frac{1}{\delta_{n}}\left\|u_{1}\right\| \cdot\left|\lambda_{2}^{\alpha}\left(B_{\lambda_{2}} u_{2}, v_{n}\right)-\lambda_{1}^{\alpha}\left(B_{\lambda_{1}} u_{1}, v_{n}\right)\right| \\
\leqq & \frac{c_{1}}{\delta_{n}}\left\|\lambda_{2}^{\alpha} B_{\lambda_{2}} u_{2}-\lambda_{1}^{\alpha} B_{\lambda_{1}} u_{1}\right\|+c_{2} \frac{\left|\mu_{n}^{\alpha}\right|}{\delta_{n}}\left\|u_{2}-u_{1}\right\|,
\end{aligned}
$$

by (3.5).

According to (3.4),

$$
\left|\lambda_{2}-\lambda_{1}\right| \leqq\left\|\lambda_{2}^{\alpha} B_{\lambda_{2}} u_{2}-\lambda_{1}^{\alpha} B_{\lambda_{1}} u_{1}\right\|
$$

But

$$
\begin{aligned}
& \left\|\lambda_{2}^{\alpha} B_{\lambda_{2}} u_{2}-\lambda_{1}^{\alpha} B_{\lambda_{1}} u_{1}\right\| \\
& \leqq\left|\lambda_{2}^{\alpha}-\lambda_{1}^{\alpha}\right| \cdot\left\|B_{\lambda_{2}} u_{2}\right\|+\left|\lambda_{1}^{\alpha}\right| \cdot\left\|\left(B_{\lambda_{2}}-B_{\lambda_{1}}\right) u_{2}\right\|+\left|\lambda_{1}^{\alpha}\right| \cdot\left\|B_{\lambda_{1}}\left(u_{2}-u_{1}\right)\right\| \\
& \leqq c_{1}\left|\mu_{n}\right|^{\alpha-1} \cdot\left|\lambda_{2}-\lambda_{1}\right|+c_{2}\left|\mu_{n}\right|^{\alpha} \cdot\left|\lambda_{2}-\lambda_{1}\right|+c_{3}\left|\mu_{n}\right|^{\alpha} \cdot\left\|u_{2}-u_{1}\right\| \text {, }
\end{aligned}
$$

by (2.4) and (3.5). Therefore,

$$
\text { (3.9) }|| \lambda_{2}^{\alpha} B_{\lambda_{2}} u_{2}-\lambda_{1}^{\alpha} B_{\lambda_{1}} u_{1}\left\|\leqq c_{1}\left|\mu_{n}\right|^{\alpha-1} \cdot\left|\lambda_{2}-\lambda_{1}\right|+c_{2}\left|\mu_{n}\right|^{\alpha} \cdot\right\| u_{2}-u_{1} \| \text {. }
$$

If $n$ is large enough, (3.8) and (3.9) together give

$$
|| \lambda_{2}^{\alpha} B_{\lambda_{2}} u_{2}-\lambda_{1}^{\alpha} B_{\lambda_{1}} u_{1}\left\|\leqq c\left|\mu_{n}^{\alpha}\right| \cdot\right\| u_{2}-u_{1} \| .
$$

Then, by (3.7),

$$
\left\|u_{2}^{\prime}-u_{1}^{\prime}\right\| \leqq c\left|\frac{\mu_{n}^{\alpha}}{\delta_{n}}\right| \cdot\left\|u_{2}-u_{1}\right\|,
$$

proving that (3.6) is a contraction if $n$ is large enough.

With this fact established, we can go on to prove the first part of the theorem. For each $n,(3.6)$ has (exactly) one fixed point, which we shall denote by $u_{n}$. Clearly, 


$$
\left(u_{n}, v_{n}\right)=1 \text {, }
$$

so that $\left(B_{n} u_{n}-\left(B_{n} u_{n}, v_{n}\right) u_{n}, v_{n}\right)=0$, and

$$
\begin{aligned}
\left(\mu_{n}-A\right) u_{n} & =\lambda_{n}^{\alpha} \sum_{j}\left(B_{n} u_{n}-\left(B_{n} u_{n}, v_{n}\right) u_{n}, v_{j}\right) v_{j} \\
& =\lambda_{n}^{\alpha} B_{n} u_{n}-\lambda_{n}^{\alpha}\left(B_{n} u_{n}, v_{n}\right) u_{n}=\lambda_{n}^{\alpha} B_{n} u_{n}-\left(\lambda_{n}-\mu_{n}\right) u_{n},
\end{aligned}
$$

by (3.4). Therefore, $u_{n}$ is an eigenfunction of (1.3), again if $n$ is large enough. The corresponding eigenvalues approach zero by (3.5).

To prove the rest of the theorem, consider

$$
\begin{aligned}
\left\|u_{n}-v_{n}\right\|^{2} & =\sum_{j \neq n}\left|\frac{\lambda_{n}^{\alpha}}{\mu_{n}-\mu_{j}}\left(B_{n} u_{n}-\left(B_{n} u_{n}, v_{n}\right) u_{n}, v_{j}\right)\right|^{2} \\
& \leqq c\left|\frac{\mu_{n}^{\alpha}}{\delta_{n}}\right|^{2} \cdot\left\|B_{n} u_{n}-\left(B_{n} u_{n}, v_{n}\right) u_{n}\right\|^{2} \leqq c\left|\frac{\mu_{n}^{\alpha}}{\delta_{n}}\right|^{2},
\end{aligned}
$$

since $u_{n} \in b_{\gamma}$. Thus, by (2.2),

$$
\sum_{N}^{\infty}\left\|u_{n}-v_{n}\right\|^{2}<1
$$

if $N$ is large enough.

All the assumptions we have made on the size of $n$ (or $N$ ) have amounted to assumptions that $\left|\mu_{n}\right|$, or $\left|\mu_{n}^{\alpha} / \delta_{n}\right|$, or $\sum_{n}^{\infty}\left|\mu_{N}^{\alpha} / \delta_{n}\right|^{2}$ are small enough. Since $\left|\mu_{1}\right|$, the largest eigenvalue of $A$, is equal to $\|A\|$, we can say that if both $\|A\|$ and $\sum_{1}^{\infty}\left|\mu_{n}^{\alpha} / \delta_{n}\right|^{2}$ are small enough, (3.11) will hold even with $N=1$. In that case, let $\left\{a_{n}\right\}$ be any finite sequence of complex numbers. Then,

$$
\begin{aligned}
\left\|\sum a_{n}\left(u_{n}-v_{n}\right)\right\|^{2} & \leqq\left(\sum\left\|u_{n}-v_{n}\right\|^{2}\right)\left(\sum\left|a_{n}\right|^{2}\right) \\
& \leqq \theta \sum\left|a_{n}\right|^{2},
\end{aligned}
$$

where $\theta<1$. Therefore, by a theorem of Paley and Wiener [2], the sequence $\left\{u_{n}\right\}$ is complete in $H$. This proves the last sentence of the theorem.

To prove (2.5), a little more is needed. Choose $N$ so that (3.11) holds, and define $P$ as the projection operator on $H_{N}$, so that

$$
P x=\sum_{N}^{\infty}\left(x, v_{j}\right) v_{j}
$$

for any $x$ in $H$. If $n \geqq N$, 


$$
P u_{n}-v_{n}\|=\| P\left(u_{n}-v_{n}\right)\|\leqq\| u_{n}-v_{n} \|
$$

so that

$$
\sum_{N}^{\infty}\left\|P u_{n}-v_{n}\right\|^{2}<1
$$

Since $\left\{P u_{n}\right\}_{N}^{\infty} \subset H_{N}$, we conclude that this sequence is complete in $H_{N}$, again by the theorem of Paley and Wiener.

Let $x \in H$. Then,

$$
\begin{aligned}
x & =P x+(I-P) x \\
& =\sum_{N}^{\infty} \xi_{n} P u_{n}+(I-P) x,
\end{aligned}
$$

for some sequence $\left\{\xi_{n}\right\}$ of complex numbers. However, the last equation can be written

$$
x=\sum_{N}^{\infty} \xi_{n} u_{n}+(I-P)\left(x-\sum_{N}^{\infty} \xi_{n} u_{n}\right),
$$

and (2.5) follows from this. This completes the proof of the theorem.

4. We shall now consider, very briefly, an application to the differential equation (1.1) in the important case when

$$
L u=\frac{d}{d x}\left(p \frac{d u}{d x}\right) .
$$

Recalling that the parameter $\lambda$ of (1.2) is the reciprocal of the $\lambda$ in (1.1), we shall call $\mu$ an "eigenvalue" of $L$ if $L u=(1 / \mu) u$. It is known [3, pp. $270 \mathrm{ff}$.] that as $n \rightarrow \infty$, the "eigenvalues" of an operator (4.1) are ultimately simple and are asymptotic to a multiple of $1 / n^{2}$. Thus, $\mu_{n} \sim c / n^{2}$, so that $\delta_{n} \sim c / n^{3}$. Therefore,

$$
\sum\left|\frac{\mu_{n}^{2}}{\delta_{n}}\right|^{2} \leqq c \sum \frac{1}{n^{2}}<\infty,
$$

and the theorem of $\$ 2$ can be applied.

\section{REFERENCES}

1. S. Chandrasekhar, Hydrodynamic stability, Clarendon Press, Oxford, 1961.

2. F. Riesz and B. Sz.-Nagy, Functional analysis, Ungar, New York, 1955.

3. E. L. Ince, Ordinary differential equations, Dover, New York, 1944.

University OF Chicago 